\title{
An easy preparation of pyridinium $N$-heteroarylaminides
}

\author{
M. José Reyes, Carolina Burgos, M. Luisa Izquierdo and Julio Alvarez-Builla* \\ Departamento de Química Orgánica, Universidad de Alcalá, Campus Universitario, Alcalá de Henares, 28871 Madrid, Spain
}

Received 1 April 2003; revised 25 July 2003; accepted 25 November 2003

Abstract-Differently substituted pyridinium $N$-heteroarylaminides have been prepared in one step with good yield from $\mathrm{N}$-aminopyridinium iodide and the corresponding heteroaryl chloride.

(C) 2003 Elsevier Ltd. All rights reserved.

For many years conjugated heterocyclic $N$-ylides, a subgroup of mesomeric betaines, ${ }^{1}$ have been widely used as building blocks for the synthesis of fused heterocyclic systems and natural products, due to its 1,3-dipolar character, ${ }^{2}$ that allows cycloaddition processes to take place efficiently. Today, cycloimmonium ylides are involved in a wide range of synthetically useful reactions, mainly in the field of heterocyclic chemistry. ${ }^{3}$

The chemistry of pyridinium $N$-(2'-heteroaryl)aminides $\mathbf{5}$ (Scheme 1) has been developed in the last few years taking advantage of its structure, in which a positively charged pyridinium ring coexists with a 2-aminoheteroaryl negatively charged moiety. This peculiar structure would selectively direct the attacking electrophiles, thus allowing easy and selective halogenations on the heteroaryl moiety. ${ }^{4-6}$ Moreover, N-alkylation process takes place regioselectively over the aminide nitrogen, by the partial blockage of the heteroaryl $\alpha$-nitrogen, via an intramolecular hydrogen bond. ${ }^{4}$ The $\mathrm{N}-\mathrm{N}$ bond reduction of the resulting pyridinium salts should allow the preparation of the corresponding amines ${ }^{4,7}$ or polyamines. ${ }^{8}$ When $\mathrm{N}$-alkylation was performed with $\alpha$-haloesters or $\alpha$-haloketones, pyrido[1,2-a]pyrimidin-4-ones and imidazo[1,2-a]pyridines were respectively obtained by a cascade heterocyclisation process. $^{9}$ Finally, intramolecular radical arylations of $\mathrm{N}$-haloazinylpyridinium $\mathrm{N}$-aminides afforded dipyridopyrazole and pyridopyrazolepyrazine derivatives. ${ }^{10}$

The synthesis of $N$-aminide intermediates $\mathbf{5}$ has been traditionally performed by attack of the corresponding 2-heteroaryl hydrazine 2 to 2,4-dinitrophenyl pyridinium chloride 1 (see Scheme 1) generating the hydrazone $\mathbf{3}$, which is again closed to a pyridinium derivative by acid catalysis, to produce the salt $\mathbf{4}$ and from there, by treatment with base, the $N$-aminides were obtained. This method, adapted from the scheme described by Beyer ${ }^{11}$ is suitable for available heteroaryl derivatives, usually the simpler pyridylhydrazines.

Alternatively, an easy method can be used for various $\pi$-deficient heterocycles, by simply treating the $N$-aminopyridinium iodide $\mathbf{6}^{12}$ with the corresponding 2-chloroheteroaryls $\mathbf{8}$ in the presence of base. The method generates the pyridinium $\mathrm{N}$-aminides $\mathbf{5}$ in only one step, and makes a suitable alternative to prepare a diversity of those useful intermediates. The methodology has been applied to the synthesis of $\mathrm{N}$-vinyl, ${ }^{13} \mathrm{~N}$-imidoyl ${ }^{14}$ and fluorinated $N$-aromatic iminopyridinium ylides. ${ }^{15}$

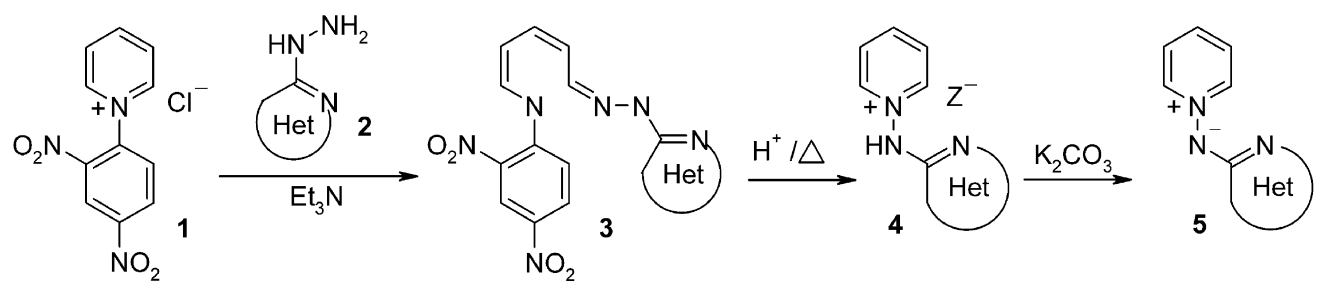

Scheme 1.

Keywords: Pyridinium $N$-aminides; $N$-ylides.

* Corresponding author. Tel.: +34-91-885-46-06; fax: +34-91-885-46-86; e-mail address: julio.alvarez@uah.es 


\section{Results and discussion}

Different $\alpha$-chloroheterocycles $\mathbf{8 a}-\mathbf{o}$ were reacted with the $N$-iminopyridinium ylide 7, generated at room temperature from the readily available $\mathrm{N}$-aminopyridinium iodide $\mathbf{6}^{12}$ and anhydrous potassium carbonate in acetonitrile (Scheme 2), to produce the corresponding pyridinium $N$-heteroarylaminides 5a-o. A set of commercial 2-(and 4-)chloropyrimidines, 2-chloropyrazines and 3-chloropyridazines, were chosen, having additional chlorine atoms on the heterocyclic ring, in order to obtain new halogenated ylides as useful starting materials in our in course studies on radical $^{10}$ processes and palladium catalysed reactions. ${ }^{16}$

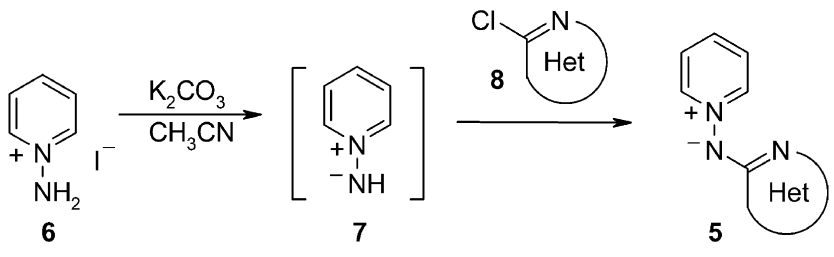

Scheme 2.
The nucleophilic substitution process works well using simpler halodiazines such as 2-chloropyrimidine and 2-chloropyrazine, yielding aminides such as $\mathbf{5 g}$ and $\mathbf{5 m}$, in only $4 \mathrm{~h}$ and with improved yields (95 and 72) in comparison over those obtained using the traditional ANRORC process, followed by treatment with base ${ }^{4}$ (72 and $64 \%$, see Table 1). However, with 2-chloropyridine the reaction did not take place, and after $48 \mathrm{~h}$ reflux, only the starting materials were recovered. As in other $\mathrm{S}_{\mathrm{N}} \mathrm{Ar}$ reactions, electron-withdrawing groups make the heterocyclic ring of the electrophile more $\pi$-deficient, facilitating the attack of the nucleophile. In this way, good yields in $N$-(pyridin-2'-yl)pyridinium aminides were obtained with 2,6-dichloropyridine, 3-nitro and 5-nitrochloropyridines and almost quantitative yields employing dichlorodiazines (see Table 1). The worse result was obtained for compound 5e, having a phenyl group, which probably increases the electronic density in the diazine ring, making the $S_{N} A r$ process more difficult. A considerable improvement was also obtained in the synthesis of $N$-( $2^{\prime}$-benzothiazol- $2^{\prime}$ yl)pyridinium aminide $\mathbf{5 p}$, previously reported in a $45 \%$ yield. ${ }^{11}$

In conclusion, we describe herein a mild and efficient method to prepare a series of pyridinium $N$-heteroarylaminides 5 in good to excellent yields from the suitable $\alpha$-haloheterocycle $\mathbf{8}$ and a stoichiometric amount of

Table 1. Compounds 5a-p obtained

\begin{tabular}{|c|c|c|c|c|}
\hline Compound & Het & Reaction time (h) & Reaction temperature & Yield (\%) \\
\hline $5 \mathbf{a}$ & & 4 & Reflux & 65 \\
\hline $5 \mathbf{b}$ & & 24 & Room temp. & 70 \\
\hline $5 c$ & & 4 & Room temp. & $75^{\mathrm{a}}$ \\
\hline $5 d$ & & 15 & Room temp. & 91 \\
\hline $5 e$ & & 4 & Reflux & 53 \\
\hline $5 f$ & & 10 & Reflux & 77 \\
\hline $5 g$ & & 4 & Reflux & $95^{\mathrm{b}}$ \\
\hline
\end{tabular}


Table 1 (continued)

\begin{tabular}{|c|c|c|c|c|}
\hline Compound & Het & Reaction time (h) & Reaction temperature & Yield $(\%)$ \\
\hline $5 i$ & & 20 & Room temp. & 85 \\
\hline $5 \mathbf{j}$ & & 7 & Room temp. & 75 \\
\hline $5 \mathbf{k}$ & & 24 & Room temp. & 98 \\
\hline 51 & & 24 & Room temp. & 88 \\
\hline $5 \mathrm{~m}$ & & 4 & Reflux & $72^{b}$ \\
\hline $5 n$ & & 20 & Room temp. & 95 \\
\hline 50 & & 20 & Room temp. & 77 \\
\hline $5 p$ & & 24 & Reflux & $90^{\mathrm{b}}$ \\
\hline
\end{tabular}

a This compound was obtained by nitration of $N$-(pyridin- $2^{\prime}$-yl)pyridinium aminide in $25 \%$ yield. ${ }^{4}$

b These compounds were obtained by the classical procedure (see Scheme 1) in $72 \%(\mathbf{5 g}), 64 \%(\mathbf{5 m})$, and $45 \%(\mathbf{5 p})$ overall yield. ${ }^{11}$

commercial 1-aminopyridinium iodide 6. This one-step procedure represents a clear improvement to previously described methodologies.

\section{Experimental}

All melting points were determined in open capillary tubes, on a Gallenkamp MFB-595-010M and are uncorrected. IR spectra were obtained on a Perkin-Elmer FTIR 1725X spectrometer. ${ }^{1} \mathrm{H}$ and ${ }^{13} \mathrm{C}$ NMR spectra were recorded on a Varian Unity 300 or $500 \mathrm{MHz}$ spectrometer at room temperature. Chemical shifts are given in $\operatorname{ppm}(\delta)$ downfield from TMS. Coupling constants $(J)$ are in hertz $(\mathrm{Hz})$, and signals are described as follows: s, singlet; d, doublet; t, triplet; br., broad; m, multiplet; ap., apparent etc. The assignment of proton and carbon resonances has been made on the basis of double resonance and two-dimensional H,Ccorrelated experiments; HSQC and HMBC spectra have been recorded when necessary on a Varian Mercury VX300 NMR System. Elemental analyses were carried out on a Heraeus Rapid CHN analyzer and are within $0.4 \%$ of the theoretical values for all new compounds described. All reagents were obtained from commercial sources and used without further purification. Solvents used were purified and dried by standard procedures. Column chromatography was carried out with silica gel $60(40-63 \mu \mathrm{m}$, Merck) columns or Biotage Flash (KP-Sil, $60 \AA$, 32-63 $\mu \mathrm{M}$ ) cartridges, using the eluent reported for each case.

\subsection{Preparation of pyridinium aminides 5}

General method. Potassium carbonate (1.86 g, $13.5 \mathrm{mmol})$ was added to a solution of the $N$-aminopyridinium iodide 6 $(1 \mathrm{~g}, 4.5 \mathrm{mmol})$ in acetonitrile $(20 \mathrm{~mL})$ and the reaction mixture was vigorously stirred for $45 \mathrm{~min}$ at room temperature to give a purple solution of compound 7 . Over this solution, the corresponding $\alpha$-chloroheterocycle $\mathbf{8}$ $(4.7 \mathrm{mmol})$ in acetonitrile $(5 \mathrm{~mL})$ was added. The mixture was either stirred at room temperature or refluxed (see Table 1) until no starting material was detected by TLC. The inorganic salts were filtered through zelite, the filtrate was evaporated in vacuo and the product was purified by chromatography on silica gel using ethanol as eluent, crystallized from the suitable solvent and identified.

The following compounds were prepared according the general method. 
2.1.1. $N$-( $6^{\prime}$-Chloropyridin- $2^{\prime}$-yl $)$ pyridinium aminide (5a). Yellow solid (601 mg, 65\%, ethanol-ethyl acetate), mp 110-112 ${ }^{\circ} \mathrm{C}$. Anal. calcd for $\mathrm{C}_{10} \mathrm{H}_{8} \mathrm{ClN}_{3}$ : C, 58.41; $\mathrm{H}$, 3.92; N, 20.43. Found: C, 58.68; H, 4.02; N, 20.44; $\nu_{\max }$ (KBr) $1586,1430,767 \mathrm{~cm}^{-1} ; \delta_{\mathrm{H}}\left(500 \mathrm{MHz} . \mathrm{CD}_{3} \mathrm{OD}\right) 8.73$ $(2 \mathrm{H}, \mathrm{dd}, J=7.1,1.2 \mathrm{~Hz}, H 2(6)) ; 8.03(1 \mathrm{H}, \mathrm{tt}, J=7.7,1.2 \mathrm{~Hz}$, $H 4) ; 7.80(2 \mathrm{H}, \mathrm{dd}, J=7.7,7.1 \mathrm{~Hz}, H 3(5)) ; 7.27(1 \mathrm{H}, \mathrm{dd}$, $\left.J=8.4,7.3 \mathrm{~Hz}, H 4^{\prime}\right) ; 6.34\left(1 \mathrm{H}, \mathrm{d}, J=8.4 \mathrm{~Hz}, H 3^{\prime}\right.$ or $\left.5^{\prime}\right) ; 6.32$ $\left(1 \mathrm{H}, \mathrm{d}, J=7.3 \mathrm{~Hz}, H 3^{\prime}\right.$ or $\left.5^{\prime}\right) ; \delta_{\mathrm{C}}\left(125 \mathrm{MHz} . \mathrm{CD}_{3} \mathrm{OD}\right) 165.7$ $\left(C 2^{\prime}\right), 149.5\left(C 6^{\prime}\right), 144.6(C 2(6)), 140.1\left(C 4^{\prime}\right), 138.2(C 4)$, $128.3(C 3(5)), 110.1\left(C 3^{\prime}\right.$ or $\left.5^{\prime}\right), 109.4\left(C 3^{\prime}\right.$ or $\left.5^{\prime}\right)$.

2.1.2. $\boldsymbol{N}$-( $3^{\prime}$-Nitropyridin- $2^{\prime}$-yl)pyridinium aminide $(5 \mathrm{~b})$. Deep orange solid (681 mg, 70\%, methanol), mp 257$258{ }^{\circ} \mathrm{C}$. Anal. calcd for $\mathrm{C}_{10} \mathrm{H}_{8} \mathrm{~N}_{4} \mathrm{O}_{2}: \mathrm{C}, 55.56 ; \mathrm{H}, 3.73 ; \mathrm{N}$, 25.91. Found: C, 55.66; H, 3.77; N, 25.66; $\nu_{\max }(\mathrm{KBr}) 1602$, $1543,1479,1426,1241 \mathrm{~cm}^{-1} ; \delta_{\mathrm{H}}\left(300 \mathrm{MHz} . \mathrm{CD}_{3} \mathrm{OD}\right) 8.63$ $(2 \mathrm{H}, \mathrm{dd}, J=6.8,1.3 \mathrm{~Hz}, H 2(6)) ; 8.30(1 \mathrm{H}, \mathrm{dd}, J=8.1,1.8 \mathrm{~Hz}$, $\left.H 4^{\prime}\right) ; 8.25(1 \mathrm{H}, \mathrm{tt}, J=7.7,1.3 \mathrm{~Hz}, H 4) ; 7.93(3 \mathrm{H}, \mathrm{m}, H 3(5)$ and $\left.6^{\prime}\right) ; 6.40\left(1 \mathrm{H}, \mathrm{dd}, J=8.1,4.6 \mathrm{~Hz}, H 5^{\prime}\right) ; \delta_{\mathrm{C}}(75 \mathrm{MHz}$. DMSO-d $\left.d_{6}\right) 158.3\left(C 2^{\prime}\right), 154.1\left(C 6^{\prime}\right), 144.1(C 2(6)), 138.8$ (C4), $135.2\left(C 4^{\prime}\right), 127.5(C 3(5)), 127.3\left(C 3^{\prime}\right), 107.6\left(C 5^{\prime}\right)$.

2.1.3. $N$ - $\left(5^{\prime}\right.$-Nitropyridin- $2^{\prime}$-yl $)$ pyridinium aminide $(5 \mathrm{c})$. Orange solid $\left(729 \mathrm{mg}, 75 \%\right.$, methanol), mp $220-222{ }^{\circ} \mathrm{C}$ (lit. $\left.{ }^{4} 216-218{ }^{\circ} \mathrm{C}\right) ; \nu_{\max }(\mathrm{KBr}) 1597,1547,1488,1426$, $1275 \mathrm{~cm}^{-1} ; \delta_{\mathrm{H}}\left(300 \mathrm{MHz} . \mathrm{CD}_{3} \mathrm{OD}\right) 8.69(2 \mathrm{H}, \mathrm{dd}, J=7.0$, $1.3 \mathrm{~Hz}, H 2(6)) ; 8.61\left(1 \mathrm{H}, \mathrm{d}, J=2.7 \mathrm{~Hz}, H 6^{\prime}\right) ; 8.26(1 \mathrm{H}$, tt, $J=7.7,1.3 \mathrm{~Hz}, H 4) ; 7.98\left(1 \mathrm{H}, \mathrm{dd}, J=9.5,2.7 \mathrm{~Hz}, H 4^{\prime}\right) ; 7.94$ $(2 \mathrm{H}, \mathrm{dd}, J=7.7,7.0 \mathrm{~Hz}, H 3(5)) ; 6.40(1 \mathrm{H}, \mathrm{d}, J=9.5 \mathrm{~Hz}$, $\left.H 3^{\prime}\right) ; \delta_{\mathrm{C}}\left(75 \mathrm{MHz} . \mathrm{DMSO}-d_{6}\right) 165.6\left(C 2^{\prime}\right), 147.9\left(C 6^{\prime}\right)$, 143.3 (C2(6)), $139.4(C 4), 131.3\left(C 5^{\prime}\right), 130.5\left(C 4^{\prime}\right), 127.4$ (C3(5)), $109.4\left(C 3^{\prime}\right)$.

2.1.4. $N$-( $6^{\prime}$-Chloropyridazin $-3^{\prime}$-yl $)$ pyridinium aminide (5d). Yellow solid (845 mg, 91\%, ethanol-ethyl acetate), mp $154-155^{\circ} \mathrm{C}$. Anal. calcd for $\mathrm{C}_{9} \mathrm{H}_{7} \mathrm{ClN}_{4}$ : C, 52.31; $\mathrm{H}$, 3.41; N, 27.11. Found: C, 52.09; H, 3.26; N, 27.13; $\nu_{\max }$ (KBr) 1577, 1474, 1414, 1341, $1149 \mathrm{~cm}^{-1} ; \delta_{\mathrm{H}}(300 \mathrm{MHz}$. $\left.\mathrm{CD}_{3} \mathrm{OD}\right) 8.79$ (2H, dd, $\left.J=6.9,1.3 \mathrm{~Hz}, H 2(6)\right) ; 8.22(1 \mathrm{H}, \mathrm{tt}$, $J=7.8,1.3 \mathrm{~Hz}, H 4) ; 7.93(2 \mathrm{H}, \mathrm{dd}, J=7.8,6.9 \mathrm{~Hz}, H 3(5))$; $7.18\left(1 \mathrm{H}, \mathrm{d}, J=9.4 \mathrm{~Hz}, H 5^{\prime}\right) ; 6.83\left(1 \mathrm{H}, \mathrm{d}, J=9.4 \mathrm{~Hz}, H 4^{\prime}\right)$; $\delta_{\mathrm{C}}\left(75 \mathrm{MHz} . \mathrm{CD}_{3} \mathrm{OD}\right) 164.9\left(C 3^{\prime}\right), 145.6(C 2(6)), 144.9$ $\left(C 6^{\prime}\right), 140.3(C 4), 129.9\left(C 5^{\prime}\right), 128.9(C 3(5)), 122.3\left(C 4^{\prime}\right)$.

2.1.5. $N$-(6'-Phenylpyridazin- $3^{\prime}$-yl)pyridinium aminide (5e). Yellow solid (592 mg, 53\%, ethanol-ethyl acetate), mp $160-161{ }^{\circ} \mathrm{C}$. Anal. calcd for $\mathrm{C}_{15} \mathrm{H}_{12} \mathrm{~N}_{4}$ : C, 72.56; $\mathrm{H}$, 4.87; N, 22.57. Found: C, 72.27; H, 4.87; N, 22.28; $\nu_{\max }$ (KBr) 1592, 1474, 1449, 1412, 1337, $1153 \mathrm{~cm}^{-1} ; \delta_{\mathrm{H}}$ (300 MHz. CD 3 OD) $8.82(2 \mathrm{H}, \mathrm{dd}, J=6.9,1.3 \mathrm{~Hz}, H 2(6))$; $8.11(1 \mathrm{H}, \mathrm{tt}, J=7.7,1.3 \mathrm{~Hz}, H 4) ; 7.85(2 \mathrm{H}, \mathrm{dd}, J=7.7$, $6.9 \mathrm{~Hz}, H 3(5)) ; 7.80\left(2 \mathrm{H}, \mathrm{dd}, J=8.2,1.5 \mathrm{~Hz}, H 2^{\prime \prime}\left(6^{\prime \prime}\right)\right) ; 7.62$ $\left(1 \mathrm{H}, \mathrm{d}, J=9.5 \mathrm{~Hz}, H 5^{\prime}\right) ; 7.40(2 \mathrm{H}, \mathrm{dd}, J=8.2,6.9 \mathrm{~Hz}$, $\left.H 3^{\prime \prime}\left(5^{\prime \prime}\right)\right) ; 7.36\left(1 \mathrm{H}, \mathrm{tt}, J=6.9,1.5 \mathrm{~Hz}, H 4^{\prime \prime}\right) ; 6.85(1 \mathrm{H}, \mathrm{d}$, $\left.J=9.5 \mathrm{~Hz}, H 4^{\prime}\right) ; \delta_{\mathrm{C}}\left(75 \mathrm{MHz} . \mathrm{CD}_{3} \mathrm{OD}\right) 165.0\left(C 3^{\prime}\right), 151.4$ $\left(C 6^{\prime}\right), 145.9(C 2(6)), 139.9(C 4), 138.8\left(C 1^{\prime \prime}\right), 130.1$ $\left(C 3^{\prime \prime}\left(5^{\prime \prime}\right)\right), 129.7\left(C 4^{\prime \prime}\right), 129.0(C 3(5)), 127.5\left(C 5^{\prime}\right), 127.0$ $\left(C 2^{\prime \prime}\left(6^{\prime \prime}\right)\right), 119.8\left(C 4^{\prime}\right)$.

2.1.6. $N$-( $4^{\prime}$-Chlorophthalazin- $\mathbf{1}^{\prime}$-yl $)$ pyridinium aminide (5f). Yellow solid (889 mg, 77\%, ethanol-ethyl acetate), mp $157-159{ }^{\circ} \mathrm{C}$. Anal. calcd for $\mathrm{C}_{13} \mathrm{H}_{9} \mathrm{ClN}_{4}$ : C, 60.83; H,
3.53; N, 21.83. Found: C, 60.64; H, 3.69; N, 21.73; $\nu_{\max }$ (KBr) 1512, 1483, 1406, 1359, $1138 \mathrm{~cm}^{-1} ; \delta_{\mathrm{H}}(300 \mathrm{MHz}$. $\left.\mathrm{CD}_{3} \mathrm{OD}\right) 8.85$ (2H, dd, $\left.J=6.9,1.3 \mathrm{~Hz}, H 2(6)\right) ; 8.40(1 \mathrm{H}, \mathrm{m}$, $H 5^{\prime}$ or $\left.H 8^{\prime}\right) ; 8.26(1 \mathrm{H}, \mathrm{tt}, J=7.8,1.3 \mathrm{~Hz}, H 4) ; 8.01(1 \mathrm{H}, \mathrm{m}$, $H 5^{\prime}$ or $\left.H 8^{\prime}\right) ; 7.96(2 \mathrm{H}, \mathrm{dd}, J=7.8,6.9 \mathrm{~Hz}, H 3(5) ; 7.87(2 \mathrm{H}$, $\mathrm{m}, H 6^{\prime}$ and $\left.H 7^{\prime}\right) ; \delta_{\mathrm{C}}\left(75 \mathrm{MHz} . \mathrm{CD}_{3} \mathrm{OD}\right) 162.0\left(C 1^{\prime}\right), 146.2$ (C2(6)), $143.5\left(C 4^{\prime}\right), 140.6(C 4), 133.3\left(C 6^{\prime}\right.$ or $\left.C 7^{\prime}\right), 133.0$ $\left(C 6^{\prime}\right.$ or $\left.C 7^{\prime}\right), 129.0(C 3(5)), 127.8\left(C 4^{\prime} \mathrm{a}\right), 125.3\left(C 5^{\prime}\right.$ or $\left.C 8^{\prime}\right)$, $125.2\left(C 5^{\prime}\right.$ or $\left.C 8^{\prime}\right), 124.4\left(C 8^{\prime} a\right)$.

2.1.7. $N$-(Pyrimidin-2' -yl)pyridinium aminide (5g). Yellow solid $(735 \mathrm{mg}, 95 \%$, ethyl acetate), mp 151$153{ }^{\circ} \mathrm{C}$ (lit. $\left.{ }^{4} 150-152{ }^{\circ} \mathrm{C}\right) ; \delta_{\mathrm{H}}\left(300 \mathrm{MHz} . \mathrm{CD}_{3} \mathrm{OD}\right) 8.71$ $(2 \mathrm{H}, \mathrm{dd}, J=6.9,1.3 \mathrm{~Hz}, H 2(6)) ; 8.20(1 \mathrm{H}, \mathrm{tt}, J=7.8,1.3 \mathrm{~Hz}$, $H 4) ; 8.11\left(2 \mathrm{H}, \mathrm{d}, J=4.8 \mathrm{~Hz}, H 4^{\prime}\left(6^{\prime}\right)\right) ; 7.91(2 \mathrm{H}, \mathrm{dd}, J=7.8$, $6.9 \mathrm{~Hz}, H 3(5)) ; 6.38\left(1 \mathrm{H}, \mathrm{t}, J=4.8 \mathrm{~Hz}, H 5^{\prime}\right) ; \delta_{\mathrm{C}}(75 \mathrm{MHz}$. $\left.\mathrm{CD}_{3} \mathrm{OD}\right) 169.4\left(C 2^{\prime}\right), 159.2\left(C 4^{\prime}(6)^{\prime}\right), 145.8(C 2(6)), 139.9$ (C4), 128.8 (C3(5)), $109.0\left(C 5^{\prime}\right)$.

2.1.8. $N$-( $2^{\prime}$-Chloropyrimidin- $4^{\prime}$-yl)pyridinium aminide (5h). Yellow solid (883 mg, 95\%, ethanol-ethyl acetate), mp $134-136{ }^{\circ} \mathrm{C}$. Anal. calcd for $\mathrm{C}_{9} \mathrm{H}_{7} \mathrm{ClN}_{4}$ : C, 52.31; H, 3.41; N, 27.11. Found: C, 52.32; H, 3.52; N, 27.26; $\nu_{\max }$ (KBr) 1618, 1587, 1474, 1335, $977 \mathrm{~cm}^{-1} ; \delta_{\mathrm{H}}(300 \mathrm{MHz}$. $\left.\mathrm{CD}_{3} \mathrm{OD}\right) 8.65(2 \mathrm{H}, \mathrm{dd}, J=6.9,1.4 \mathrm{~Hz}, H 2(6)) ; 8.27(1 \mathrm{H}, \mathrm{tt}$, $J=7.6,1.4 \mathrm{~Hz}, H 4) ; 7.94(2 \mathrm{H}, \mathrm{dd}, J=7.6,6.9 \mathrm{~Hz}, H 3(5))$; $7.65\left(1 \mathrm{H}, \mathrm{d}, J=6.2 \mathrm{~Hz}, H 6^{\prime}\right) ; 6.25\left(1 \mathrm{H}, \mathrm{br} . \mathrm{d}, J=6.2 \mathrm{~Hz}, H 5^{\prime}\right)$; $\delta_{\mathrm{C}}\left(75 \mathrm{MHz} . \mathrm{CD}_{3} \mathrm{OD}\right) 168.9\left(C 4^{\prime}\right), 160.8\left(C 2^{\prime}\right), 153.8\left(C 6^{\prime}\right)$, $145.2(C 2(6)), 141.2(C 4), 128.6(C 3(5)), 105.8\left(C 5^{\prime}\right)$.

2.1.9. $N$-(6'-Chloropyrimidin- $\mathbf{4}^{\prime}$-yl)pyridinium aminide (5i). Yellow solid (790 mg, 85\%, ethanol-ethyl acetate), mp 167- $168{ }^{\circ} \mathrm{C}$. Anal. calcd for $\mathrm{C}_{9} \mathrm{H}_{7} \mathrm{ClN}_{4}$ : C, 52.31; H, 3.41; N, 27.11. Found: C, 52.47; H, 3.47; N, 27.01; $\nu_{\max }$ $(\mathrm{KBr}) 1619,1574,1448,1336,1066,974 \mathrm{~cm}^{-1} ; \delta_{\mathrm{H}}$ (300 MHz. CD 3 OD) $8.66(2 \mathrm{H}, \mathrm{dd}, J=6.9,1.4 \mathrm{~Hz}, H 2(6))$; $8.26(1 \mathrm{H}, \mathrm{tt}, J=7.8,1.4 \mathrm{~Hz}, H 4) ; 7.92(2 \mathrm{H}, \mathrm{dd}, J=7.8$, $6.9 \mathrm{~Hz}, H 3(5)) ; 7.81\left(1 \mathrm{H}, \mathrm{s}, H 2^{\prime}\right) ; 6.30\left(1 \mathrm{H}\right.$, br.s, $\left.H 5^{\prime}\right) ; \delta_{\mathrm{C}}$ (75 MHz. CD 3 OD) $168.9\left(C 4^{\prime}\right), 158.6\left(C 2^{\prime}\right), 156.7\left(C 6^{\prime}\right)$, $145.2(C 2(6)), 141.1(C 4), 128.6(C 3(5)), 104.5\left(C 5^{\prime}\right)$.

2.1.10. $N$-(2' $2^{\prime}, 6^{\prime}$-Dichloropyrimidin- $4^{\prime}-$ yl $)$ pyridinium aminide (5j). Yellow solid (814 mg, 75\%, ethanol-ethyl acetate), $\mathrm{mp}>190{ }^{\circ} \mathrm{C}$, dec. Anal. calcd for $\mathrm{C}_{9} \mathrm{H}_{6} \mathrm{Cl}_{2} \mathrm{~N}_{4}$ : C, 44.84; H, 2.51; N, 23.24. Found: C, 45.15; H, 2.64; N, $23.06 ; \nu_{\max }(\mathrm{KBr}) 1618,1574,1460,1391,1146,989 \mathrm{~cm}^{-1}$; $\delta_{\mathrm{H}}\left(300 \mathrm{MHz} . \mathrm{CD}_{3} \mathrm{OD}\right) 8.69(2 \mathrm{H}, \mathrm{dd}, J=6.9,1.3 \mathrm{~Hz}$, $H 2(6)) ; 8.33(1 \mathrm{H}, \mathrm{tt}, J=7.8,1.3 \mathrm{~Hz}, H 4) ; 7.99(2 \mathrm{H}, \mathrm{dd}$, $J=7.8,6.9 \mathrm{~Hz}, H 3(5)) ; 6.30\left(1 \mathrm{H}\right.$, br.s, $\left.H 5^{\prime}\right) ; \delta_{\mathrm{C}}(75 \mathrm{MHz}$. $\left.\mathrm{CD}_{3} \mathrm{OD}\right) \quad 169.7\left(C 4^{\prime}\right), 160.5\left(C 2^{\prime}\right), 156.9\left(C 6^{\prime}\right), 145.2$ (C2(6)), 141.8 (C4), $128.9(C 3(5)), 103.4\left(C 5^{\prime}\right)$.

2.1.11. $N$-(2'-Chloro-6 ${ }^{\prime}$-methylpyrimidin- $4^{\prime}$-yl $)$ pyridinium aminide (5k). Yellow solid (972 mg, 98\%, hexane-ethyl acetate), mp $144-146{ }^{\circ} \mathrm{C}$. Anal. calcd for $\mathrm{C}_{10} \mathrm{H}_{9} \mathrm{ClN}_{4}$ : C, 54.43; H, 4.11; N, 25.39. Found: C, 54.11; $\mathrm{H}, \quad 4.03 ; \mathrm{N}, 25.17 ; \nu_{\max }(\mathrm{KBr}) 1597,1457,1267$, $1177 \mathrm{~cm}^{-1} ; \delta_{\mathrm{H}}\left(300 \mathrm{MHz} . \mathrm{CD}_{3} \mathrm{OD}\right) 8.68(2 \mathrm{H}, \mathrm{dd}, J=6.9$, $1.3 \mathrm{~Hz}, H 2(6)) ; 8.30(1 \mathrm{H}, \mathrm{tt}, J=7.8,1.3 \mathrm{~Hz}, H 4) ; 7.97(2 \mathrm{H}$, dd, $J=7.8,6.9 \mathrm{~Hz}, H 3(5)) ; 6.13\left(1 \mathrm{H}\right.$, br.s, $\left.H 5^{\prime}\right) ; 2.21(3 \mathrm{H}, \mathrm{s}$, $\left.\mathrm{CH}_{3}\right) ; \delta_{\mathrm{C}}\left(75 \mathrm{MHz} . \mathrm{CD}_{3} \mathrm{OD}\right) 169.6\left(\mathrm{C}^{\prime}\right), 164.3\left(\mathrm{C6}^{\prime}\right), 160.5$ $\left(C 2^{\prime}\right), 145.3(C 2(6)), 141.1(C 4), 128.7(C 3(5)), 103.4\left(C 5^{\prime}\right)$; $22.8\left(\mathrm{CH}_{3}\right)$. 
2.1.12. $N$-( $6^{\prime}$ - Chloro- $2^{\prime}$-methylsulfanylpyrimidin $-4^{\prime}$ yl)pyridinium aminide (5l). Yellow solid (998 mg, 88\%, hexane-ethyl acetate), mp $158-160{ }^{\circ} \mathrm{C}$. Anal. calcd for $\mathrm{C}_{10} \mathrm{H}_{9} \mathrm{ClN}_{4} \mathrm{~S}$ : C, 47.53; H, 3.59; N, 22.17; S, 12.69. Found: C, 47.30; H, 3.51; N, 22.03; S, $12.35 \nu_{\max }(\mathrm{KBr}) 1617,1541$, $1447,1369,1214 \mathrm{~cm}^{-1} ; \delta_{\mathrm{H}}\left(300 \mathrm{MHz} . \mathrm{CD}_{3} \mathrm{OD}\right) 8.72(2 \mathrm{H}$, $\mathrm{dd}, J=7.0,1.3 \mathrm{~Hz}, H 2(6)) ; 8.31(1 \mathrm{H}, \mathrm{tt}, J=7.8,1.3 \mathrm{~Hz}, H 4)$; $7.97(2 \mathrm{H}, \mathrm{dd}, J=7.8,7.0 \mathrm{~Hz}, H 3(5)) ; 6.06\left(1 \mathrm{H}, \mathrm{br} . \mathrm{s}, H 5^{\prime}\right)$; $2.08\left(3 \mathrm{H}, \mathrm{s}, \mathrm{CH}_{3}\right) ; \delta_{\mathrm{C}}\left(75 \mathrm{MHz} . \mathrm{CD}_{3} \mathrm{OD}\right) 172.2\left(\mathrm{C}^{\prime}\right), 168.4$ $\left(C 2^{\prime}\right), 156.9\left(C 6^{\prime}\right), 145.8(C 2(6)), 141.3(C 4), 128.5(C 3(5))$, $99.6\left(\mathrm{C5}^{\prime}\right) ; 13.7\left(\mathrm{CH}_{3}\right)$.

2.1.13. $\quad \boldsymbol{N}$-(Pyrazin-2'-yl)pyridinium aminide $(\mathbf{5 m})$. Yellow solid $(557 \mathrm{mg}, 72 \%$, ethyl acetate), mp 158$159{ }^{\circ} \mathrm{C}$ (lit. $\left.{ }^{4} 157-159{ }^{\circ} \mathrm{C}\right) ; \delta_{\mathrm{H}}\left(300 \mathrm{MHz} . \mathrm{CD}_{3} \mathrm{OD}\right) 8.82$ $(2 \mathrm{H}, \mathrm{dd}, J=7.0,1.2 \mathrm{~Hz}, H 2(6)) ; 8.21(1 \mathrm{H}, \mathrm{tt}, J=7.8,1.2 \mathrm{~Hz}$, $H 4) ; 7.93(2 \mathrm{H}, \mathrm{dd}, J=7.8,7.0 \mathrm{~Hz}, H 3(5)) ; 7.86(1 \mathrm{H}, \mathrm{d}$, $\left.J=1.5 \mathrm{~Hz}, H 3^{\prime}\right) ; 7.61\left(1 \mathrm{H}, \mathrm{dd}, J=3.1,1.5 \mathrm{~Hz}, H 6^{\prime}\right) ; 7.45$ $\left(1 \mathrm{H}, \mathrm{d}, J=3.1 \mathrm{~Hz}, H 5^{\prime}\right) ; \delta_{\mathrm{C}}\left(75 \mathrm{MHz} . \mathrm{CD}_{3} \mathrm{OD}\right) 162.0\left(C 2^{\prime}\right)$, $145.3(C 2(6)), 141.9\left(C 6^{\prime}\right), 139.9(C 4), 136.8\left(C 3^{\prime}\right), 129.5$ $\left(C 5^{\prime}\right), 128.9(C 3(5))$.

2.1.14. $N$-( $3^{\prime}$-Chloropyrazin- $2^{\prime}$-yl $)$ pyridinium aminide (5n). Yellow solid ( $883 \mathrm{mg}, 95 \%$, ethanol-ethyl acetate), mp 197-199 ${ }^{\circ} \mathrm{C}$. Anal. calcd for $\mathrm{C}_{9} \mathrm{H}_{7} \mathrm{ClN}_{4}$ : C, 52.31; H, $3.41 ; \mathrm{N}, 27.11$. Found: C, 52.25; H, 3.52; N, 27.13; $\nu_{\max }$ (KBr) 1612, 1559, 1474, 1443, 1397, $1045 \mathrm{~cm}^{-1} ; \delta_{\mathrm{H}}$ (300 MHz. CD $\left.{ }_{3} \mathrm{OD}\right) 8.68(2 \mathrm{H}, \mathrm{dd}, J=6.8,1.3 \mathrm{~Hz}, H 2(6))$; $8.22(1 \mathrm{H}, \mathrm{tt}, J=7.7,1.3 \mathrm{~Hz}, H 4) ; 7.91(2 \mathrm{H}, \mathrm{dd}, J=7.7$, $6.8 \mathrm{~Hz}, H 3(5)) ; 7.49\left(1 \mathrm{H}, \mathrm{d}, J=2.8 \mathrm{~Hz}, H 6^{\prime}\right) ; 7.21(1 \mathrm{H}, \mathrm{d}$, $\left.J=2.8 \mathrm{~Hz}, H 5^{\prime}\right) ; \delta_{\mathrm{C}}\left(75 \mathrm{MHz} . \mathrm{CD}_{3} \mathrm{OD}\right) 159.7\left(C 2^{\prime}\right), 146.4$ $(C 2(6)), 141.2\left(C 6^{\prime}\right), 140.9(C 4), 136.6\left(C 3^{\prime}\right), 129.2(C 3(5))$, $128.1\left(C 5^{\prime}\right)$.

2.1.15. $N$-( $6^{\prime}$-Chloropyrazin- $2^{\prime}$ yl $)$ pyridinium aminide (5o). Yellow solid $(715 \mathrm{mg}, 77 \%$, ethanol-ethyl acetate), mp $184-186{ }^{\circ} \mathrm{C}$. Anal. calcd for $\mathrm{C}_{9} \mathrm{H}_{7} \mathrm{ClN}_{4}$ : C, 52.31; H, 3.41; N, 27.11. Found: C, 52.45; H, 3.47; N, 27.15; $\nu_{\max }$ (KBr) 1618, 1555, 1465, 1445, 1389, $984 \mathrm{~cm}^{-1} ; \delta_{\mathrm{H}}$ (300 MHz. CD $\left.{ }_{3} \mathrm{OD}\right) 8.78(2 \mathrm{H}, \mathrm{dd}, J=6.9,1.3 \mathrm{~Hz}, H 2(6))$; $8.21(1 \mathrm{H}, \mathrm{tt}, J=7.8,1.3 \mathrm{~Hz}, H 4) ; 7.93(2 \mathrm{H}, \mathrm{dd}, J=7.8$, $6.9 \mathrm{~Hz}, H 3(5)) ; 7.70\left(1 \mathrm{H}, \mathrm{s}, H 3^{\prime}\right) ; 7.36\left(1 \mathrm{H}, \mathrm{s}, H 5^{\prime}\right) ; \delta_{\mathrm{C}}$ (75 MHz. $\left.\mathrm{CD}_{3} \mathrm{OD}\right) 161.9\left(C 2^{\prime}\right), 147.8\left(C 6^{\prime}\right), 145.1(C 2(6))$, $140.1(C 4), 134.0\left(C 3^{\prime}\right), 128.7(C 3(5)), 126.0\left(C 5^{\prime}\right)$.

2.1.16. $N$-(Benzothiazol-2' - yl)pyridinium aminide (5p). Yellow solid (919 mg, 90\%, hexane-ethyl acetate), mp $170-172{ }^{\circ} \mathrm{C}$ (lit. $\left.{ }^{11} 167-169{ }^{\circ} \mathrm{C}\right) ; \delta_{\mathrm{H}}\left(300 \mathrm{MHz} . \mathrm{CD}_{3} \mathrm{OD}\right)$ $9.07(2 \mathrm{H}, \mathrm{dd}, J=6.9,1.3 \mathrm{~Hz}, H 2(6)) ; 8.16(1 \mathrm{H}, \mathrm{tt}, J=7.8$, $1.3 \mathrm{~Hz}, H 4) ; 7.92(2 \mathrm{H}, \mathrm{dd}, J=7.8,6.9 \mathrm{~Hz}, H 3(5)) ; 7.52(1 \mathrm{H}$, $\left.\mathrm{dd}, J=7.8,1.3 \mathrm{~Hz}, H 7^{\prime}\right) ; 7.22\left(1 \mathrm{H}, \mathrm{dd}, J=8.0,1.6 \mathrm{~Hz}, H 4^{\prime}\right)$; $7.17\left(1 \mathrm{H}, \mathrm{ddd}, J=8.0,7.0,1.3 \mathrm{~Hz}, H 5^{\prime}\right) ; 6.97(1 \mathrm{H}, \mathrm{ddd}$, $\left.J=7.8,7.0,1.6 \mathrm{~Hz}, H 6^{\prime}\right) ; \delta_{\mathrm{C}}\left(75 \mathrm{MHz} . \mathrm{CD}_{3} \mathrm{OD}\right) 176.9\left(C 2^{\prime}\right)$, $153.8\left(C 3^{\prime} \mathrm{a}\right), 143.9(C 2(6)), 139.2(C 4), 131.5\left(C 7^{\prime} \mathrm{a}\right), 128.7$ $(C 3(5)), 126.4\left(C 5^{\prime}\right), 121.7\left(C 6^{\prime}\right), 121.4\left(C 7^{\prime}\right), 117.8\left(C 4^{\prime}\right)$.

\section{Acknowledgements}

The authors wish to thank the Ministerio de Ciencia y Tecnología (projects CICYT-2FD97-1248 and BQU20011508) for financial support, and the Ministerio de Educación y Cultura for a studenship (M.J.R.).

\section{References and notes}

1. Ollis, D. W.; Stanforth, S. P. Tetrahedron 1985, 41, 2239-2329.

2. 1,3-Dipolar cycloaddition chemistry; Padwa, A., Ed.; WileyInterscience: New York, 1984.

3. Vaquero, J. J.; Alvarez-Builla, J. Adv. Nitrogen Heterocycl. 2000, 4, 159-250.

4. Carceller, R.; García-Navío, J. L.; Izquierdo, M. L.; AlvarezBuilla, J.; Fajardo, M.; Gomez-Sal, P.; Gago, F. Tetrahedron 1994, 50, 4995-5012.

5. Burgos, C.; Delgado, F.; García-Navío, J. L.; Izquierdo, M. L.; Alvarez-Builla, J. Tetrahedron 1995, 51, 8649-8654.

6. García de Viedma, A.; Martínez-Barrasa, V.; Burgos, C.; Izquierdo, M. L.; Alvarez-Builla, J. J. Org. Chem. 1999, 64, 1007-1010.

7. Martínez-Barrasa, V.; Delgado, F.; Burgos, C.; García-Navío, J. L.; Izquierdo, M. L.; Alvarez-Builla, J. Tetrahedron 2000, 56, 2481-2490.

8. Reyes, M. J.; Delgado, F.; Izquierdo, M. L.; Alvarez-Builla, J. Tetrahedron 2002, 58, 8573-8579.

9. De la Rosa, R.; Martinez-Barrasa, V.; Burgos, C.; AlvarezBuilla, J. Tetrahedron Lett. 2000, 41, 5837-5840.

10. Núñez, A.; García de Viedma, A.; Martinez-Barrasa, V.; Burgos, C.; Alvarez-Builla, J. Synlett 2002, 1093-1096.

11. Beyer, H.; Thieme, E. J. Prakt. Chem. 1966, 31, 293-303.

12. Gösl, R.; Meuwesen, A. Org. Synth. 1973, 5, 43-45.

13. Sasaki, T.; Kanematsu, K.; Kakehi, A. J. Org. Chem. 1972, 37, 3106-3110.

14. Kakehi, A.; Ito, S.; Uchiyama, K.; Konno, Y.; Kondo, K. J. Org. Chem. 1977, 42, 443-448.

15. Banks, R. E.; Hitchen, S. M. J. Fluorine Chem. 1978, 12, 159-166.

16. García-Cuadrado, D.; Cuadro, A. M.; Alvarez-Builla, J.; Vaquero, J. J. Synlett 2002, 1904-1906. 